\section{Origin of condensation nuclei in the springtime polar stratosphere}

Jingxia Zhao'

Department of Atmospheric Sciences, University of California, Los Angeles

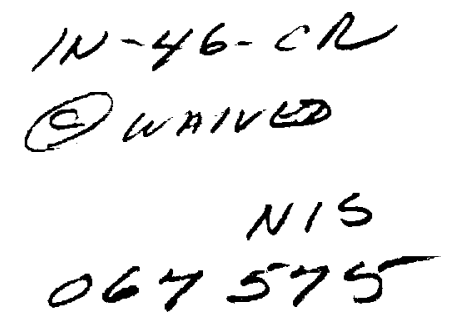

Owen B. Toon

NASA Ames Research Center, Moffett Field, California

Richard P. Turco

Department of Atmospheric Sciences, University of California, Los Angeles

\begin{abstract}
An enhanced sulfate aerosol layer has been observed near $25 \mathrm{~km}$ accompanying springtime ozone depletion in the Antarctic stratosphere. We use a onedimensional aerosol model that includes photochemistry, particle nucleation, condensational growth, coagulation, and sedimentation to study the origin of the layer. Annual cycles of sunlight, temperature, and ozone are incorporated into the model. Our results indicate that binary homogeneous nucleation leads to the formation of very small droplets of sulfuric acid and water under conditions of low temperature and production of $\mathrm{H}_{2} \mathrm{SO}_{4}$ following polar sunrise. Photodissociation of carbonyl sulfide (OCS) alone, however, cannot provide sufficient $\mathrm{SO}_{2}$ to create the observed condensation nuclei (CN) layer. When subsidence of $\mathrm{SO}_{2}$ from very high altitudes in the polar night vortex is incorporated into the model, the $\mathrm{CN}$ layer is reasonably reproduced. The model predictions, based on the subsidence in polar vortex, agree with in situ measurements of particle concentration, vertical distribution, and persistence during polar spring.
\end{abstract}

\section{Introduction}

Balloon-borne counters have been able to measure aerosols with radii greater than $0.01 \mu \mathrm{m}$ in the stratosphere since 1973 [Hofmann and Rosen, 1977; Rosen and Hofmann, 1977]. These fine particles are generally referred to as condensation nuclei $(\mathrm{CN})$ because of their small size and their role as sites for condensation of sulfuric acid vapor [Hofmann and Rosen, 1985; Hofmann, 1990]. Measurements over Antarctica have revealed an enhanced $\mathrm{CN}$ layer between 20 and $30 \mathrm{~km}$ with a maximum particle concentration exceeding $100 \mathrm{~cm}^{-3}$ at about $25 \mathrm{~km}$ [Hofmann and Rosen, 1985; Hofmann, 1988; Wilson et al., 1989]. The layer has been observed to appear repeatedly in each spring from September to November. Hofmann et al. [1989] found that the $\mathrm{CN}$ layer and the severe ozone depletion (ozone hole) in the south polar region are concurrent, and both $\mathrm{CN}$ concentrations and ozone abundance are sensitive to local temperature. Moreover, coincident with the $\mathrm{CN}$ layer and the ozone hole is an observed decrease in concentration of "optical-active aerosols" (OA) with particle radii greater then $0.15 \mu \mathrm{m}$. Recent measurements in the Arctic during winter show a similar layering effect [Hofmann, 1990]. Stratospheric $\mathrm{CN}$, which are most likely liquid sulfuric acid and water solutions, are believed to serve as sites for

\footnotetext{
'Now at Department of Meteorology, School of Ocean and Earth Science and Technology, University of Hawaii, Honolulu.

Copyright 1995 by the American Geophysical Union.

Paper number 94JD03110.

0148-0227/95/94JD-03110\$05.00
}

heterogeneous chemical reactions. Thus it is of great interest to investigate the mechanisms which lead to the formation of these $\mathrm{CN}$ layers.

Several mechanisms for the formation of the $\mathrm{CN}$ layer have been proposed. Rosen and Hofmann [1983] suggested that sulfur-bearing gases (carbonyl sulfide (OCS)) could accumulate in the polar stratosphere during winter due to the lack of sunlight and produce high concentrations of $\mathrm{H}_{2} \mathrm{SO}_{4}$ vapor in spring after sunrise. However, Oppenheimer [1987] argued that photodecomposition of OCS is a slow process because of the large zenith angle in the polar region; the production of $\mathrm{H}_{2} \mathrm{SO}_{4}$ through OCS photolysis is not sufficient for $\mathrm{CN}$ formation. Thus he proposed that photooxidation of OCS or reaction of OCS with elevated levels of $\mathrm{OH}$ might account for an enhancement in sulfuric acid vapor leading to the formation of the $\mathrm{CN}$ layer.

Because $C N$ layers are always observed to be accompanied by low temperatures, Hofmann [1988] suggested that low temperatures in the winter polar stratosphere support homogeneous nucleation and rapid growth of fine particles due to excessive supersaturation of $\mathrm{H}_{2} \mathrm{SO}_{4}$ vapor. Hamill et al. [1990] studied the homogeneous nucleation of ambient sulfuric acid at polar winter low temperatures and concluded that even with modest acid concentrations the formation of $\mathrm{H}_{2} \mathrm{SO}_{4} / \mathrm{H}_{2} \mathrm{O}$ droplets would be efficient. However, such acid concentrations may not exist in polar winter because production of $\mathrm{H}_{2} \mathrm{SO}_{4}$ through photochemical processes ceases after polar sunset, while the acid vapor still condenses to preexisting particles as temperatures are decreasing. Hamill et al. did not include the loss of $\mathrm{H}_{2} \mathrm{SO}_{4}$ to preexisting particles. Their model did not reproduce the observed sea- 
Table 1. Photochemical Reactions of Sulfur Components in the Stratosphere

\begin{tabular}{|c|c|c|}
\hline & Reaction & Rate \\
\hline$R_{1}$ & $\mathrm{~S}+\mathrm{O}_{2} \stackrel{\kappa_{1}}{\rightarrow} \mathrm{SO}+\mathrm{O}$ & $K_{1}=2.3 \times 10^{-12}$ \\
\hline$R_{2}$ & $\mathrm{SO}+\mathrm{O}_{2} \stackrel{\mathrm{K}_{2}}{\rightarrow} \mathrm{SO}_{2}+\mathrm{O}$ & $K_{2}=2.6 \times 10^{-13} e^{-2400 / T}$ \\
\hline$R_{3}$ & $\mathrm{SO}_{2}+\mathrm{OH}+\mathrm{M} \stackrel{K_{3}}{\rightarrow} \mathrm{HSO}_{3}+\mathrm{M}$ & $\begin{array}{l}K_{0}^{300}=3.0 \times 10^{-31}, n=3.3^{*} \\
K_{x}^{300}=1.5 \times 10^{-12}, m=0^{*}\end{array}$ \\
\hline$R_{4}$ & $\mathrm{HSO}_{3}+\mathrm{O}_{2} \stackrel{\kappa_{4}}{\rightarrow} \mathrm{SO}_{3}+\mathrm{HO}_{2}$ & $K_{4}=1.3 \times 10^{-12} e^{-330 / T}$ \\
\hline$R_{5}$ & $\mathrm{SO}_{3}+\mathrm{H}_{2} \mathrm{O} \stackrel{\mathrm{K} 3}{\rightarrow} \mathrm{H}_{2} \mathrm{SO}_{4}$ & $K_{S}=6.0 \times 10^{-15}+$ \\
\hline$R_{6}^{*}$ & $\mathrm{OCS}+\mathrm{O} \stackrel{K_{6}}{\rightarrow} \mathrm{SO}+\mathrm{CO}$ & $K_{6}=2.1 \times 10^{-11} e^{-2200 / T}$ \\
\hline$J_{1}$ & $\mathrm{OCS}+h v \stackrel{J_{1}}{\rightarrow} \mathrm{S}+\mathrm{CO}(\lambda<228 \mathrm{~nm})$ & \\
\hline$J_{2}$ & $\mathrm{SO}_{2}+h v \stackrel{J_{2}}{\rightarrow} \mathrm{SO}+\mathrm{O}(\lambda<220 \mathrm{~nm})$ & \\
\hline
\end{tabular}

sonal nor altitudinal characteristics of the CN layers. Rosen and Hofmann [1983] proposed that evaporation of sulfate aerosol in a stratospheric warming event followed by sufficiently rapid cooling could be responsible for the $\mathrm{CN}$ formation. However, the evaporation of aerosols may not release sufficient acid vapor for $\mathrm{CN}$ formation because the concentrations of preexisting aerosol near $30 \mathrm{~km}$ are very low.

Measurements of long-lived tracers suggest organized downward motion within the polar night vortex [Toon et al., 1989; Russell et al., 1993]. For example, data from the Halogen Occultation Experiment (HALOE) instrument on the Upper Atmosphere Research Satellite (UARS) show that methane $\left(\mathrm{CH}_{4}\right)$ mixing ratios in polar stratosphere are as low as those in the mesosphere. Moreover, the data suggest that air with lower $\mathrm{CH}_{4}$ mixing ratios in the polar vortex does not mix with the stratospheric air surrounding the vortex until the vortex breaks down in spring [Russell et al., 1993]. It can be inferred that the air at the top of the springtime polar stratosphere originates from the mesosphere by descending motion in the polar vortex associated with radiative cooling during the winter. $\mathrm{SO}_{2}$ may achieve a high mixing ratio in the mesosphere through photolysis of $\mathrm{H}_{2} \mathrm{SO}_{4}$. Although accurate measurements of the $\mathrm{H}_{2} \mathrm{SO}_{4}$ ultraviolet absorption spectrum are not available, photodissociation at wavelength below $\sim 250 \mathrm{~nm}$ is likely. $\mathrm{SO}_{2}$ generated in the mesosphere may then be transported into the stratospheric polar vortex by subsidence. There, $\mathrm{SO}_{2}$ has a relatively long lifetime because of the absence of $\mathrm{OH}$ during polar winter. In this situation the mesosphere can be considered as a sulfur source for $\mathrm{CN}$ formation in spring after sunrise. Hofmann et al. [1988] also suggested that a possible source of sulfuric acid may be transport from sunlit regions in lower latitude with entrance into the vortex above $30 \mathrm{~km}$. Then subsidence and radiative cooling in the lower stratosphere may result in particle formation.

Dust particles and micrometeorites from the ablation of micrometeors in the upper atmosphere may contribute to the CN concentrations above $20 \mathrm{~km}$ [Hunten et al., 1980; Turco et al., 1981]. These particles can affect the properties of stratospheric aerosols by serving as condensation nuclei for sulfuric acid droplets, enhancing the sulfate aerosol concentrations and reducing average particle sizes when sulfuric acid becomes supersaturated.

Polar stratospheric clouds (PSCs) may also modulate CN populations. PSCs can scavenge preexisting sulfur particles and thus decrease the total aerosol surface area in spring. The decreased aerosol surface area limits $\mathrm{H}_{2} \mathrm{SO}_{4}$ vapor loss onto the preexisting particle surfaces and maintains high $\mathrm{H}_{2} \mathrm{SO}_{4}$ vapor concentrations for homogeneous nucleation and $\mathrm{CN}$ formation.

In this study we elaborate on these various hypotheses by conducting a series of numerical experiments that include in a consistent way chemistry, aerosol microphysics, annual variations of temperature and sunlight, micrometeorite fluxes, and polar vortex dynamics.

\section{Model Description}

To simulate the behavior of $\mathrm{CN}$ in the springtime polar stratosphere, we incorporated sulfur photochemistry and microphysics in a one-dimensional version of the tracer model developed by Toon et al. [1988]. Table 1 gives the sulfur photochemical reactions which are important in the lower stratosphere. Under volcanically quiescent stratospheric conditions, the sulfur oxides originate primarily from OCS transported through the tropopause, as first suggested by Crutzen [1976]. After reaching the stratosphere, photodecomposition of $\operatorname{OCS}\left(J_{1}\right)$ releases sulfur atoms $(\mathrm{S})$, which are quickly converted into sulfur dioxide $\left(\mathrm{SO}_{2}\right)$ through $R_{1}$ and $R_{2}$. The hydroxyl $(\mathrm{OH})$ radical is critical in oxidizing $\mathrm{SO}_{2}$ to sulfuric acid $\left(\mathrm{H}_{2} \mathrm{SO}_{4}\right)$ through reactions $R_{3}$ to $R_{5}$. Because $R_{4}$ and $R_{5}$ are relatively fast, sulfuric acid production is limited mainly by reactions $J_{1}$ and $R_{3}$. In this study, the baseline profiles of $\mathrm{OH}$, $\mathrm{O}$, and $\mathrm{H}_{2} \mathrm{O}$ are assumed to be fixed with concentrations taken from the photochemical model of Brasseur and Solomon [1986]. All the chemical reaction rates correspond to standard compilations [DeMore et al., 1990].

Three types of aerosols are considered in this model: involatile (or solid) particles which originate from the troposphere (we also refer these "tropospheric" CN as Aitken particles), pure $\mathrm{H}_{2} \mathrm{SO}_{4} / \mathrm{H}_{2} \mathrm{O}$ particles, and mixed aerosols 
consisting of Aitken particles in $\mathrm{H}_{2} \mathrm{SO}_{4} / \mathrm{H}_{2} \mathrm{O}$ solutions. Aitken particles are transported from the troposphere into. the lower stratosphere and provide the core for mixed aerosols either through heterogeneous nucleation or coagulation between Aitken particles and pure $\mathrm{H}_{2} \mathrm{SO}_{4}-\mathrm{H}_{2} \mathrm{O}$ particles. Mixed aerosols interact with $\mathrm{H}_{2} \mathrm{SO}_{4}$ vapor by condensation and evaporation processes. If a mixed aerosol is completely evaporated, then its core is released and returned to the "Aitken" category. Pure $\mathrm{H}_{2} \mathrm{SO}_{4} / \mathrm{H}_{2} \mathrm{O}$ aerosols can be produced only by homogeneous nucleation, when the supersaturation of $\mathrm{H}_{2} \mathrm{SO}_{4} / \mathrm{H}_{2} \mathrm{O}$ vapor exceeds a critical value. An efficient algorithm to calculate homogeneous and heterogeneous nucleation rates [Zhao, 1993] is used here. Details concerning the other microphysical processes and corresponding numerical algorithms can be found in the works of Turco et al. [1979], Toon et al. [1988], and Zhao [1993]. In the calculations that follow, each type of particle (Aitken nuclei, pure, and mixed particles) is subdivided by size. The smallest size treated in the model is about $0.001 \mu \mathrm{m}$. The total number of particles in our simulations corresponds to the sum over the whole size bins. The observed $\mathrm{CN}$ correspond to the total number of particles with sizes greater than about $0.01 \mu \mathrm{m}$. To compare with observations, the concentration of $\mathrm{CN}$ in our simulations is by summing all the appropriate size bins in the model.

\section{Simulation Parameters}

\section{Benchmark Simulation}

There are two purposes for investigating the background aerosols before simulating transient $\mathrm{CN}$ layers. First, we need to verify the capability of the model to generate realistic vertical concentration profiles of the background aerosols and key chemical species. Second, the polar stratospheric model is initialized using the background aerosol profiles, which approximate the state of the aerosol layer in summer. To achieve a well-characterized background state, we first integrate the model to a steady state using 10-year

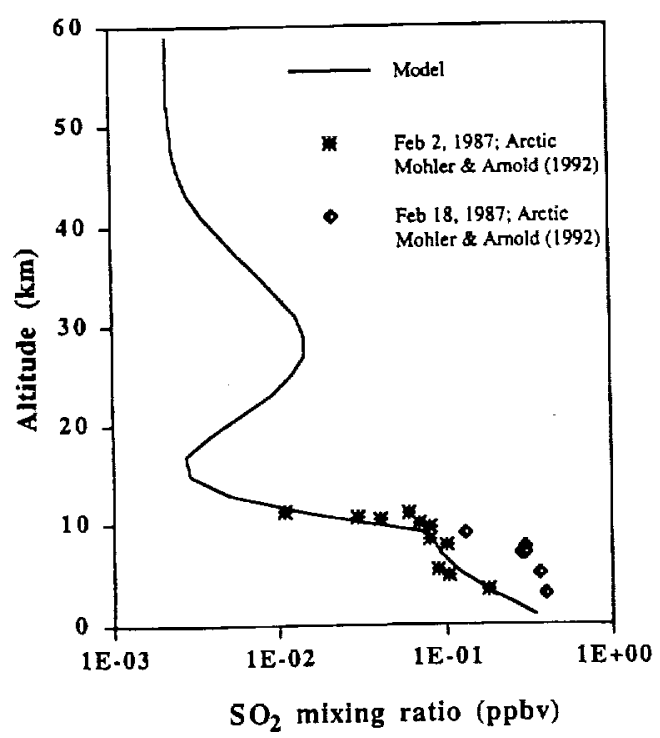

Figure 1. Calculated and observed vertical distributions of background $\mathrm{SO}_{2}$ concentrations. $\mathrm{SO}_{2}$ concentrations in the troposphere are highly variable and cannot be accurately predicted with a one-dimensional model.

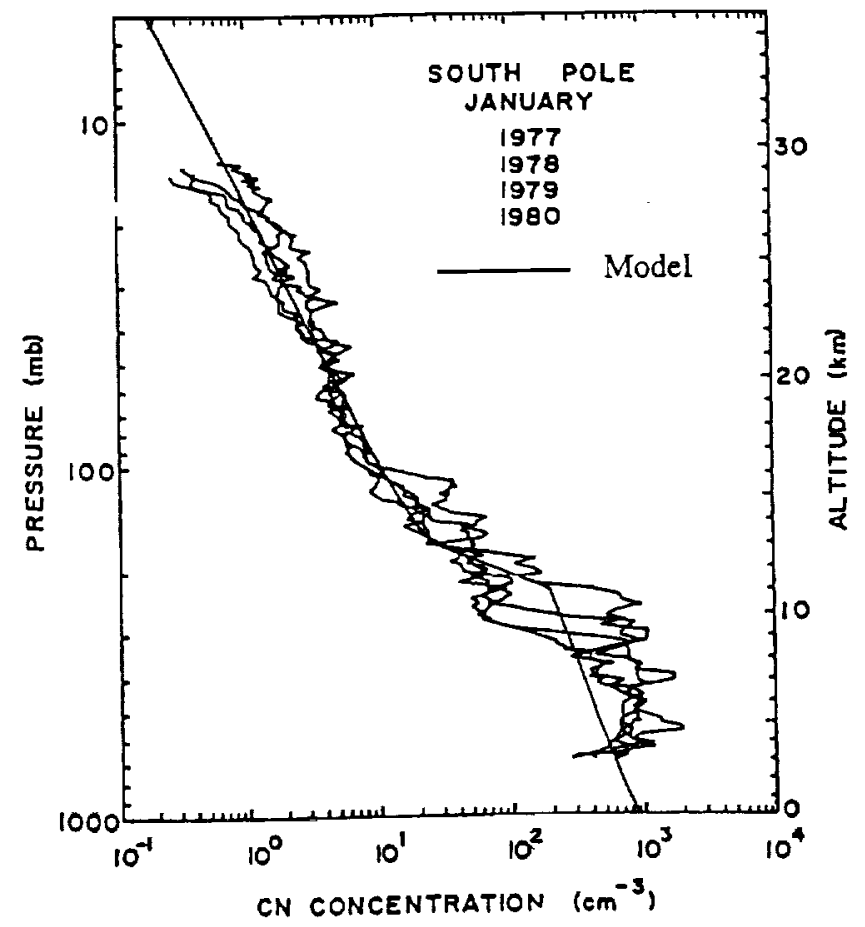

Figure 2. Calculated vertical distribution of background total aerosol concentration compared to observations [Hofmann, 1988].

mean temperatures between $70^{\circ}$ and $80^{\circ} \mathrm{S}$ and a monthly mean ozone profile. The tropopause was set at $9 \mathrm{~km}$ and was kept constant through all of the numerical experiments described here. The baseline vertical profiles of $\mathrm{SO}_{2}$ (Figure 1) and aerosol concentration (Figure 2) are reasonable comparing with observations. The computed size distributions (Figure 3) are similar to those calculated by Turco et al. [1979]. The simulated CN concentration is about $5 \mathrm{~cm}^{-3}$ at $20 \mathrm{~km}$ and less than $1 \mathrm{~cm}^{-3}$ at $30 \mathrm{~km}$; these values are close to the annual average aerosol concentrations measured near the south pole at the same heights.

\section{Annual Cycle of Temperature}

The observed high correlation between $\mathrm{CN}$ concentrations and low stratospheric temperatures suggests the importance of temperature variation in forcing the anomalous $\mathrm{CN}$. In our "polar" simulations the annual cycle of temperature is deduced from observed zonal mean temperatures between $70^{\circ}$ and $80^{\circ} \mathrm{S}$ for 1984 (Figure 4). These data present a typical picture of temperature variations in the southern polar region; similar temperatures hold in other years. In general, temperatures decrease from fall through winter and recover in the spring. The lowest temperature in Figure 4 is less than $190^{\circ} \mathrm{K}$, near $18 \mathrm{~km}$ in later winter and early spring. An approximation to the period of twilight in the polar region is superimposed on this temperature field. Near latitude of $75^{\circ} \mathrm{S}$, sunset occurs in early May and sunrise in late August. Figure 4 shows clearly that in spring after sunrise, stratospheric temperatures can remain low for a period of several weeks. This cycle of temperature and sunlight implies that the photochemical production of $\mathrm{H}_{2} \mathrm{SO}_{4}$ vapor recovers after polar sunrise, while the equilibrium vapor pressure of $\mathrm{H}_{2} \mathrm{SO}_{4}$ under low-stratospheric temperature is still very small. Therefore the acid vapor concentrations can easily 


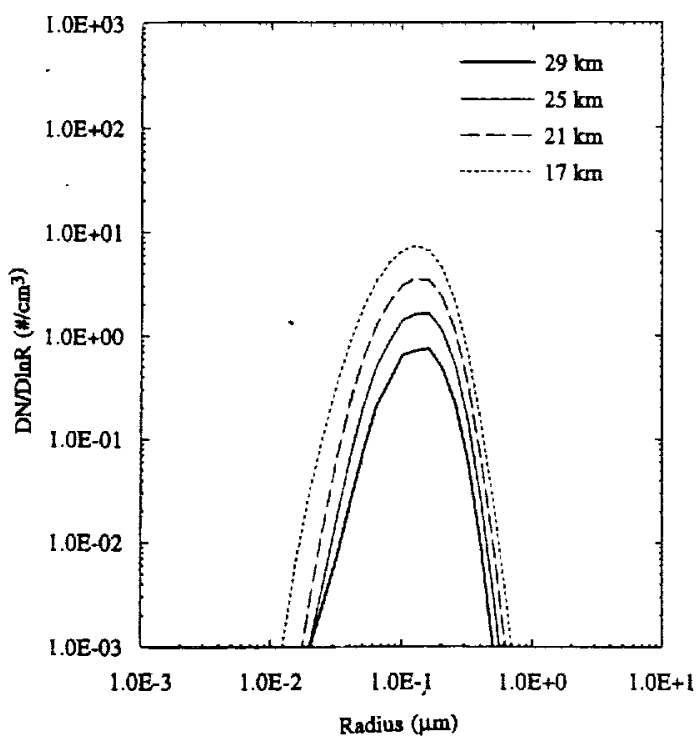

Figure 3. Calculated particle size distributions of the background aerosols at altitudes of $17,21,25$, and $29 \mathrm{~km}$. These size distributions compare favorably with measurements summarized by Turco et al. [1982].

exceed the critical value required for binary homogeneous nucleation. Then, new droplets of $\mathrm{H}_{2} \mathrm{SO}_{4} / \mathrm{H}_{2} \mathrm{O}$ solutions can be formed and grow to $\mathrm{CN}$ sizes by condensation and coagulation processes.

\section{Photolysis of Carbonyl Sulfide (OCS)}

Under ambient stratospheric conditions, sulfate aerosols originate from sulfur-bearing gases, especially OCS. Although one dimensional models are not the best tools for simulating OCS transport from the troposphere, the vertical stratification can be represented in this way. Indeed, the photodecomposition of OCS has been suggested as one cause of the CN layer [Rosen and Hofmann, 1983]. OCS photolysis is controlled by solar ultraviolet radiation, which is particularly sensitive to ozone abundances. Therefore to calculate the photodissociation rate of OCS in the southern polar region more accurately, monthly average ozone profiles derived from balloon soundings at Syowa $\left(69^{\circ} \mathrm{S}\right)$ in 1990

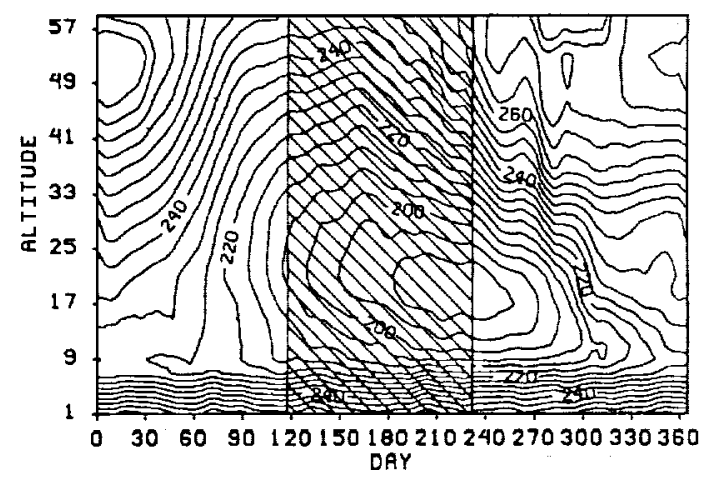

Figure 4. Isotherms of observed zonal belt-mean temperatures between $70^{\circ}$ and $80^{\circ} \mathrm{S}$ latitudes for 1984 . The data are derived from the National Meteorological Center analysis. The shaded area indicates the period of darkness during polar winter at a latitude of $75^{\circ} \mathrm{S}$. were adopted. Stratospheric ozone concentrations decline in September, reach minimum values in October, and gradually recover in November (Figure 5). These spring ozone "hole" profiles have reduced ozone abundances that favor the photodecomposition of OCS.

\section{Baseline CN Simulation}

Using the annual cycles of zonal mean temperature, solar illumination, and ozone concentrations derived from measurements in the southern polar region and the benchmark stratosphere as an initial state, a "baseline" simulation of $\mathrm{CN}$ formation was performed to investigate $\mathrm{CN}$ layer formation. This baseline model represents the best first-guess parameterization for stratospheric $\mathrm{CN}$ formation, based on existing observations and theories. In the baseline run, integration begins at day 80 , when the initial state is very close to the summer climatology and runs to day 365 , when the solution essentially returns to the background state.

The simulated $\mathrm{H}_{2} \mathrm{SO}_{4}$ mixing ratio drops dramatically as polar sunset advances at around day 120 and remains near zero until sunrise the following spring (Figure 6). The loss of $\mathrm{H}_{2} \mathrm{SO}_{4}$ vapor occurs because the normal balance between $\mathrm{H}_{2} \mathrm{SO}_{4}$ vapor production and condensation on preexisting aerosols cannot be maintained when solar illumination vanishes, and the $\mathrm{OH}$ radicals that generate $\mathrm{H}_{2} \mathrm{SO}_{4}$ disappear. In addition, decreasing temperatures favor $\mathrm{H}_{2} \mathrm{SO}_{4}$ loss onto the preexisting droplets. Below $35 \mathrm{~km}, \mathrm{H}_{2} \mathrm{SO}_{4}$ vapor reappears rapidly after sunrise on day 230 ; and photochemical reactions driven by sunlight reestablish the production of $\mathrm{H}_{2} \mathrm{SO}_{4}$. Subsequently, the $\mathrm{H}_{2} \mathrm{SO}_{4}$ mixing ratio fluctuates in accord with temperature changes. Above about $40 \mathrm{~km}$ the $\mathrm{H}_{2} \mathrm{SO}_{4}$ reduction in midwinter does not occur because of higher temperatures there that prevent condensation. Figure 7 provides several snapshots of the evolution of the vertical distribution of $\mathrm{H}_{2} \mathrm{SO}_{4}$ concentrations. During the winter period the evaporation level for sulfuric acid (i.e., where sulfuric acid vapor becomes undersaturated and evaporation can occur) rises from 37 to $45 \mathrm{~km}$ owing to the wintertime trend of temperature decrease in this region.

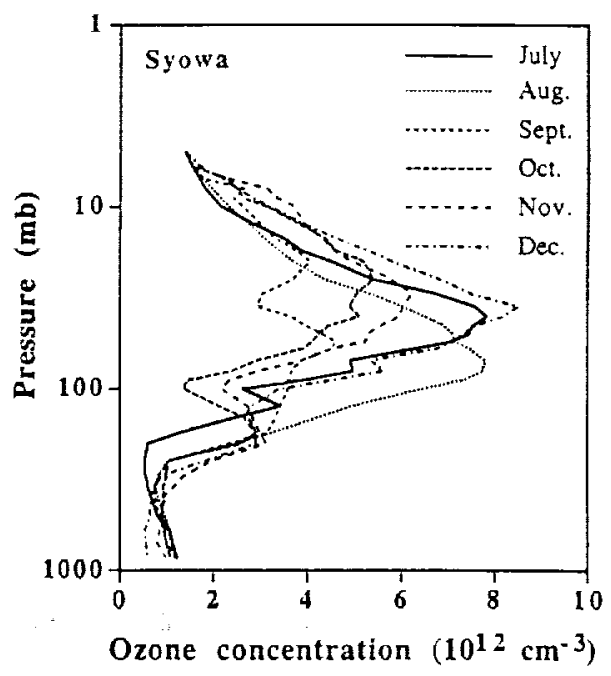

Figure 5. Monthly mean profiles of observed ozone concentrations with unit of $10^{12} \mathrm{~cm}^{-3}$ at Syowa Station $\left(69^{\circ} \mathrm{S}\right.$, $40^{\circ} \mathrm{E}$ ) in 1990 [Nankyoku and Kansokutai, 1990]. 


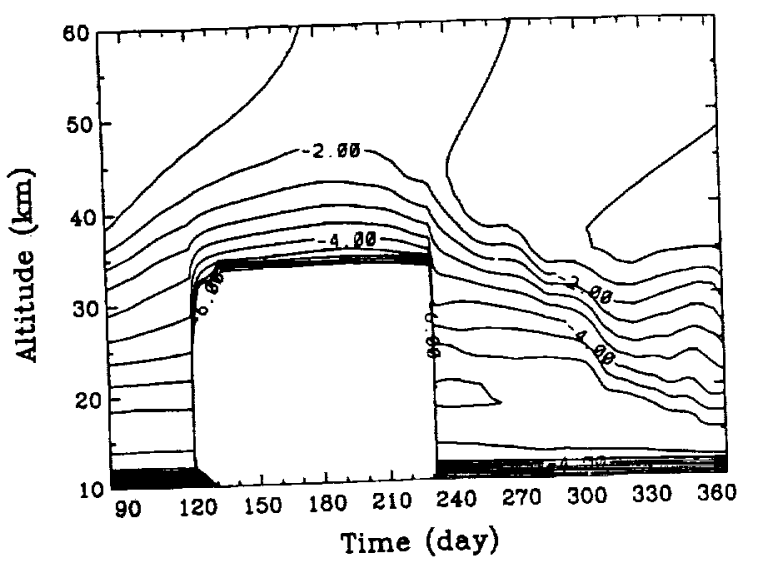

Figure 6. Variation of $\mathrm{H}_{2} \mathrm{SO}_{4}$ 'concentrations with time and altitude in the baseline condensation nuclei (CN) simulation. The logarithmic contours are identified by the power of 10 of the $\mathrm{H}_{2} \mathrm{SO}_{4}$ mixing ratio in parts per billion by volume (ppbv). The contour interval is $0.5 \log$ units.

Along with the rapid recovery of $\mathrm{H}_{2} \mathrm{SO}_{4}$ just after polar sunrise, temperatures in the lower stratosphere are near their minimum values (Figure 4). This creates an ideal situation for particle formation by nucleation, which depends on $\mathrm{H}_{2} \mathrm{SO}_{4}$ concentration and temperature. Indeed, the simulated total aerosol concentrations (all particles larger than $0.001 \mu \mathrm{m}$ ) between the tropopause and $35 \mathrm{~km}$ increase dramatically due to the new particle formation by homogeneous nucleation shortly after sunrise (Figure 8 ). The aerosol concentrations produced by homogeneous nucleation can initially exceed $10^{3} \mathrm{~cm}^{-3}$ near $25 \mathrm{~km}$. These newly formed sulfate embryos, however, cannot grow to measurable "CN" sizes. Only a few particles near $30 \mathrm{~km}$ are found reaching measurable sizes (greater than $0.01 \mu \mathrm{m}$ ) and the springtime perturbation of $\mathrm{CN}$ concentration is hardly noticeable (Figure 9). The simulated baseline $\mathrm{CN}$ profiles are not comparable to the observed $\mathrm{CN}$ layer (Figure 10). Moreover, the aerosol size distributions in the baseline

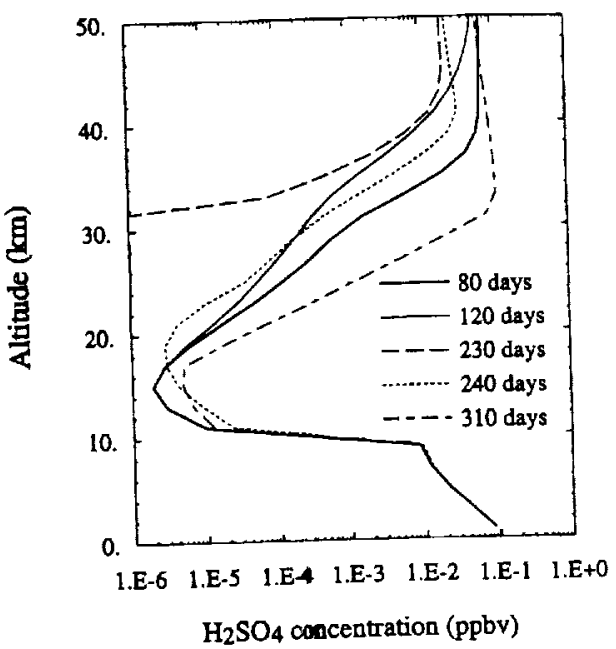

Figure 7. Vertical profiles of $\mathrm{H}_{2} \mathrm{SO}_{4}$ mixing ratio (ppbv) at different times is indicated in the figure. The concentrations correspond to the baseline $\mathrm{CN}$ simulation. Sunrise is at day 230.

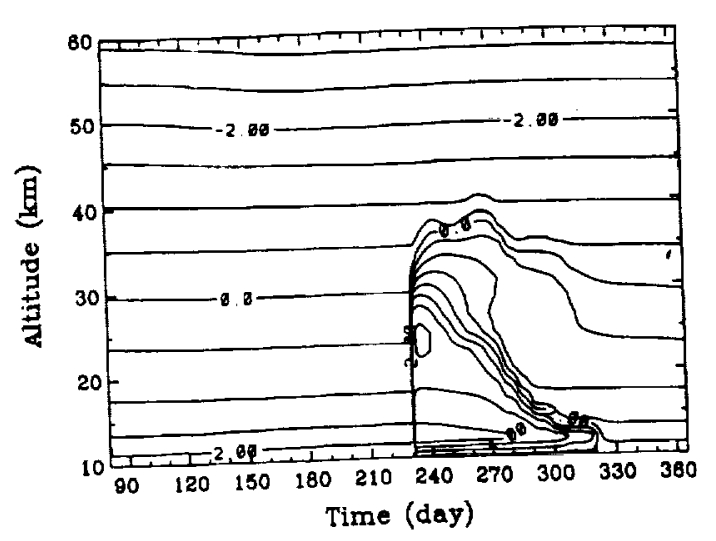

Figure 8. Variation of total aerosol concentration (number per cubic centimeter) with time and altitude in the "baseline" $\mathrm{CN}$ simulation. The concentration contours are shown with the logarithm with a power of 10 . The contour interval is $0.5 \log$ units.

experiment show that although a large number of fine $\mathrm{H}_{2} \mathrm{SO}_{4} / \mathrm{H}_{2} \mathrm{O}$ particles are initially produced by homogeneous nucleation right after sunrise, they do not grow to $\mathrm{CN}$ sizes because of a shortage of $\mathrm{H}_{2} \mathrm{SO}_{4}$ vapor and quick coagulation with ambient particles (Figure 11).

The baseline experiment fails to produce a substantial $\mathrm{CN}$ layer. The photodissociation rate of OCS is too low to produce much $\mathrm{H}_{2} \mathrm{SO}_{4}$ vapor in early spring $\left(<10^{-9} \mathrm{~s}^{-1}\right)$ owing to the weak solar illumination. Hence the conversion of ambient $\mathrm{OCS}$ into $\mathrm{H}_{2} \mathrm{SO}_{4}$ cannot be the dominant mechanism in the formation of the $\mathrm{CN}$ layer in polar spring. This is true even if the OCS concentration is allowed to accumulate through vertical diffusion over the winter. In fact, the polar vortex is highly stable against upward vertical mixing, and the vertical profiles of most long-lived tracers suggest sustained subsidence in the winter polar vortex, which should reduce OCS concentrations at $25-30 \mathrm{~km}$. It follows from the baseline simulation that certain mechanisms not treated in the baseline experiment must play an important role in providing extra sulfur for the formation of new $\mathrm{CN}$.

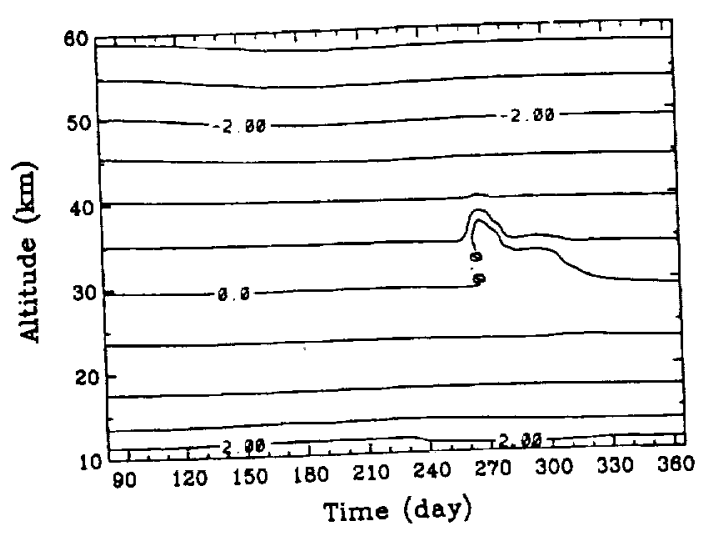

Figure 9. Variation of $\mathrm{CN}$ concentration, i.e., number of particles per cubic centimeter with radii exceeding $0.01 \mu \mathrm{m}$. The $\mathrm{CN}$ concentrations are shown as isopleths of constant concentration to logarithm. The contour interval is $0.5 \mathrm{log}$ units. 


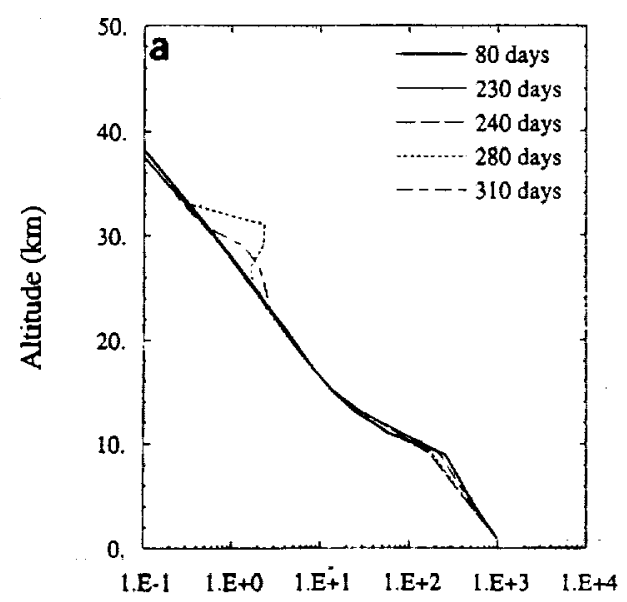

Aerosol concentration $(\mathrm{r}>0.01 \mu \mathrm{m})$

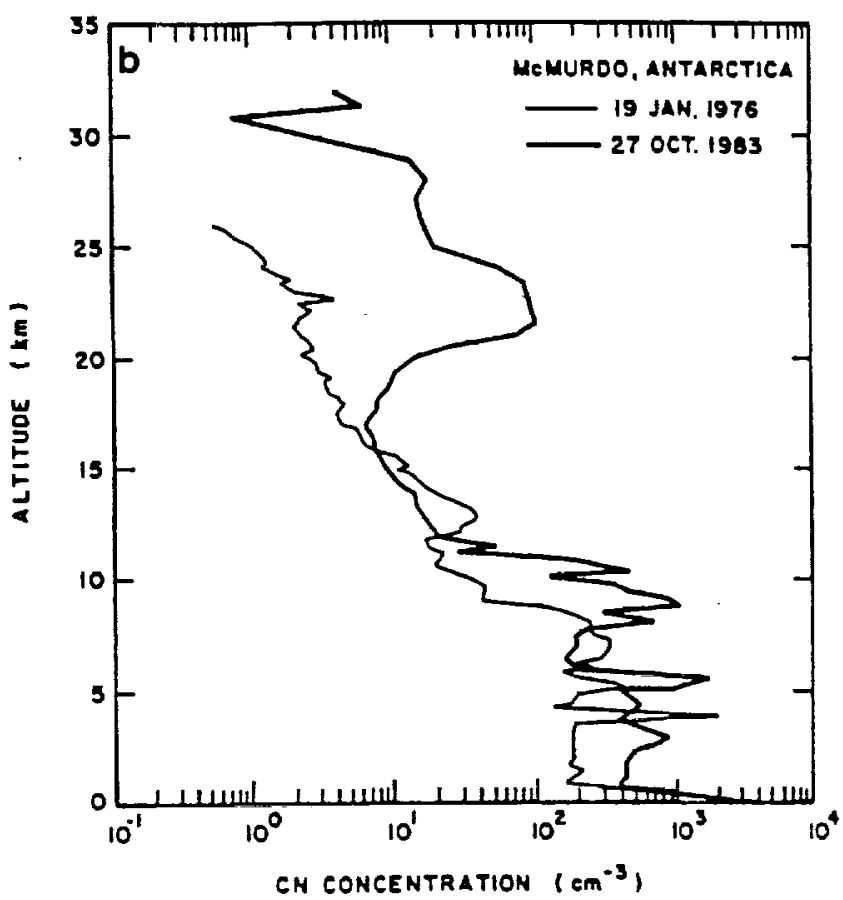

Figure 10. (a) and (b) Vertical distributions of $\mathrm{CN}$ concentrations at different times in the baseline model simulation, compared to observations [Hofmann and Rosen, 1985].

\section{Role of Subsiding Motion in the Polar Vortex}

A Lagrangian visualization using a three-dimensional model simulation provides a detailed picture of the airflow in the middle atmosphere [Fisher et al., 1993]. Air from the mesosphere executes an inward and downward spiral into the polar vortex. The numerical simulation offers an explanation of HALOE observations of low mixing ratios of methane in the polar stratosphere [Russell et al., 1993]. The subsiding motion in the polar vortex transports mesospheric air with low mixing ratios of methane down into the stratosphere. Similarly, the subsiding motion would transport a higher mixing ratio of sulfur dioxide into the polar stratosphere from the mesosphere. A high mixing ratio of $\mathrm{SO}_{2}$ in the mesosphere is likely from the photolysis of $\mathrm{H}_{2} \mathrm{SO}_{4}$ at those altitudes [Turco et al., 1979]. The $\mathrm{SO}_{2}$ transported downward would not be destroyed in the polar night because of the absence of $\mathrm{OH}$ radicals, hence the descending air can provide a critical sulfur reservoir for $\mathrm{CN}$ formation in the spring. The weak $\mathrm{CN}$ layer in the baseline experiment is thus compensated by a mesospheric sulfur source.

There are no quantitative observations of the amplitude, structure, or duration of the descending motion in the polar vortex, but three-dimensional model simulations [Fisher et al., 1993] provide useful guidelines. The model results show that the mean poleward velocity in the mesosphere is about $40^{\circ}$ per month, whereas the subsiding velocity in the upper stratosphere of the polar vortex is about $12 \mathrm{~km}$ per month in July. The descending motion decreases to about $3 \mathrm{~km}$ per month in the middle stratosphere and the poleward drift is also much slower than in the mesosphere. The vortex circulation can maintain this structure for up to 6 months.

On the basis of these model results we postulate a subsiding velocity field as

$$
w=w_{0}\left(\frac{\rho_{r}}{\rho}\right)^{\alpha} \cos \left[\pi\left(\frac{t-220}{180}\right)\right], 120<t<310 .
$$

Here $t$ is time (day), $p_{\tau}$ is the air density at the top layer of the model, $w_{0}=0.5 \mathrm{~cm} / \mathrm{s}$, and $\alpha=0.4$. This idealized formulation of vertical descent qualitatively agrees with the results of the three-dimensional simulation.

The effect of the corresponding poleward airflow is treated consistently by including an additional term in the onedimensional model to ensure mass continuity. First, note that the horizontal advection is related to the vertical velocity divergence though the mass continuity equation. Moreover, the continuity equation can be written for any tracer in the form

$$
\frac{\partial C}{\partial t}+\nabla_{H} \cdot\left(\vec{v}_{H} C\right)+\frac{\partial(w C)}{\partial z}=\nabla \cdot \rho \vec{K} \nabla\left(\frac{C}{\rho}\right)+P-L,
$$

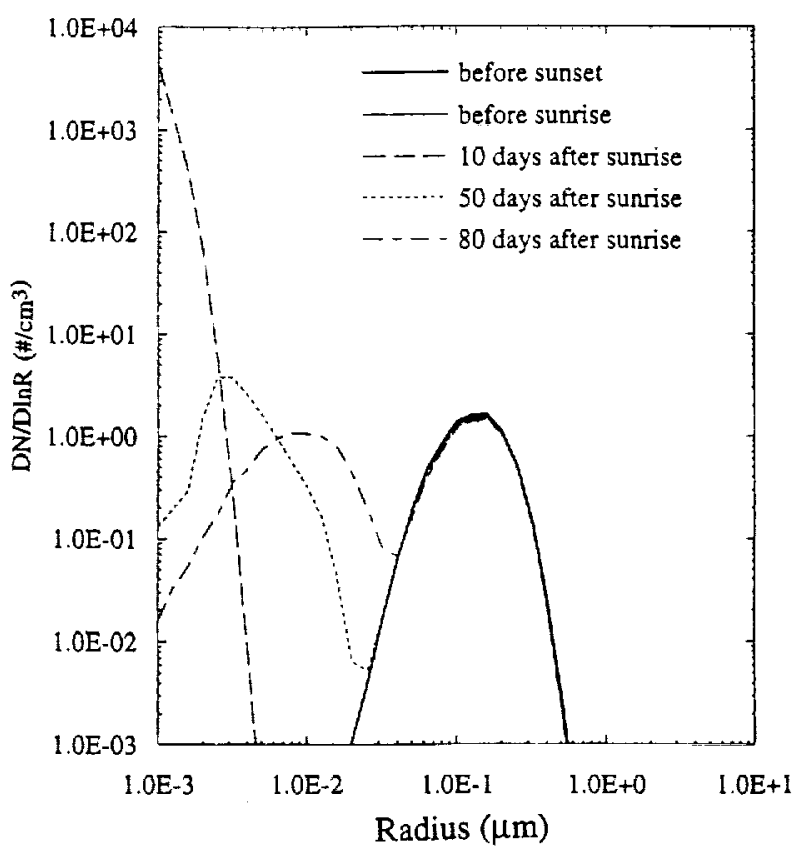

Figure 11. Aerosol size distributions at different times in the baseline model simulation. 
If zonal symmetry is assumed, the horizontal advection term can be approximated by differencing from the pole over the radius $l$ of the vortex as

$$
\nabla_{H} \cdot\left(\vec{v}_{H} C\right) \approx-\frac{v_{H} C_{a}}{l} .
$$

Here the subscript $a$ indicates values in ambient air outside the vortex at the same altitude, and $v_{H}$ is the average horizontal poleward velocity. Similarly, the mass continuity equation can be written as

$$
-\frac{v_{H}}{l}+\frac{1}{\rho} \frac{\partial(w \rho)}{\partial z}=0 .
$$

Then, in a one-dimensional tracer representation we have

$$
\frac{\partial C}{\partial t}+\frac{\partial(w C)}{\partial z}=C_{a} \frac{1}{\rho} \frac{\partial(w \rho)}{\partial z}+\frac{\partial}{\partial z} \rho K \frac{\partial}{\partial z}\left(\frac{C}{\rho}\right)+P-L
$$

The first term on the right-hand side of (5) can be viewed as the effect of horizontal mass convergence associated with net vertical subsidence. Whereas the vertical velocity used here increases with altitude, the downward mass flux actually decreases with altitude, and the horizontal convergence effectively brings air into the vortex from outside. Both the vertical motion and the horizontal convergence decrease in the lower stratosphere, and there is little accumulation of mass in the stratospheric column.

As a variation of a baseline experiment, a $\mathrm{SO}_{2}$ mixing ratio of 0.1 parts per billion by volume (ppbv) is assumed at the upper boundary $(60 \mathrm{~km})$. Similarly, the mixing ratio of $\mathrm{H}_{2} \mathrm{SO}_{4}$ in air descending from mesosphere is set to $0.1 \mathrm{ppbv}$, and the concentrations of preexisting aerosols are set to zero. These concentrations for $\mathrm{SO}_{2}$ and $\mathrm{H}_{2} \mathrm{SO}_{4}$ are taken from photochemical simulations of the stratospheric sulfur cycle [e.g., Turco et al., 1979, 1982]; however, the actual concentrations are uncertain. As will be shown, the results are not sensitive to the $\mathrm{H}_{2} \mathrm{SO}_{4}$ concentration but are sensitive to $\mathrm{SO}_{2}$.

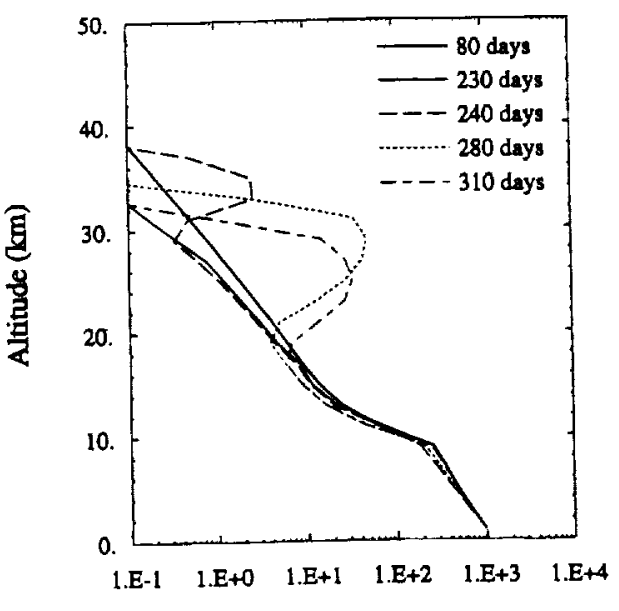

Aerosol concentration $(r>0.01 \mu \mathrm{m})$

Figure 12. Vertical distributions of $\mathrm{CN}$ concentrations at different times in the experiment including a subsiding motion from the mesosphere. Compare with data shown in Figure 10.

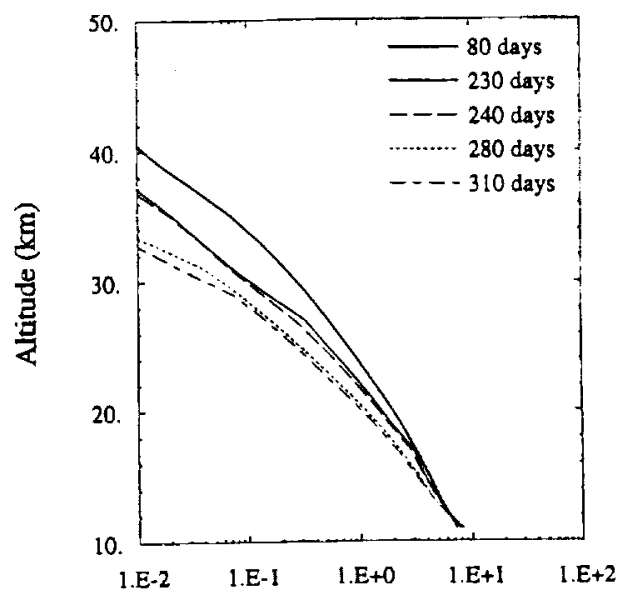

Aerosol concentration $(r>0.15 \mu \mathrm{m})$

Figure 13. The vertical distribution of large aerosol particles with $r>0.15 \mu \mathrm{m}$, versus time in the simulation with subsidence.

After including such a descending motion and associated fluxes at the upper boundary, the model generates dense $\mathrm{CN}$ layers during spring after sunrise that agree with observational data (Figure 12). The layer appears at higher altitudes in early spring and shifts downward as time progresses. The maximum concentration of $\mathrm{CN}$ particles is situated near 29 $\mathrm{km}$ in September and $24 \mathrm{~km}$ in October. The background particles in this case also have lower concentrations during winter and spring because of the downward flow, which brings particle-free air into the upper and middle stratosphere. The number of large particles is reduced significantly (Figure 13). Hence subsidence is also consistent with the aerosol hole observed by balloon-borne and satellite soundings [Hofmann and Rosen, 1983; Mc Cormick et al., 1983].

The vertical flux of $\mathrm{SO}_{2}$ at upper boundary is crucial to forming the $\mathrm{CN}$ layer. During fall the photolysis of OCS decreases and the $\mathrm{SO}_{2}$ mixing ratio above $20 \mathrm{~km}$ decreases through reaction with $\mathrm{OH}$. The photochemical source of $\mathrm{SO}_{2}$ remains insignificant throughout the winter. Moreover, the production of $\mathrm{SO}_{2}$ from $\mathrm{OCS}$ is too slow to generate a $\mathrm{CN}$ layer in the springtime. Once the downward transport of $\mathrm{SO}_{2}$ from the mesosphere into the stratospheric vortex is included, however, the $\mathrm{SO}_{2}$ mixing ratio can be maintained at an elevated level, as shown in Figure 14.

Variations in the $\mathrm{H}_{2} \mathrm{SO}_{4}$ mixing ratios for the case with subsidence are similar to those in Figure 6, except the mixing ratio of $\mathrm{H}_{2} \mathrm{SO}_{4}$ has a higher value near $30 \mathrm{~km}$ in winter and early spring due to the subsidence motion; however, the total aerosol and $\mathrm{CN}$ concentrations (Figures 15 and 16, respectively) are considerably different from those in Figures 8 and 9 . The perturbations in the aerosol concentrations following sunrise are far more intense and persistent. Note particularly the strong enhancement in $\mathrm{CN}$ concentrations from 18 to $30 \mathrm{~km}$ during springtime in Figure 16. By comparing Figures 15 and 16 , it becomes evident that the enhancement in CN concentrations occurs mainly above 18 $\mathrm{km}$, although many small droplets are generated below this level (these very small particles are not counted as $\mathrm{CN}$ ). At the lower altitudes the ambient aerosols capture all of the small droplets by coagulation before they grow to $\mathrm{CN}$ sizes by condensation. The vertical distribution of the $\mathrm{CN}$ con- 


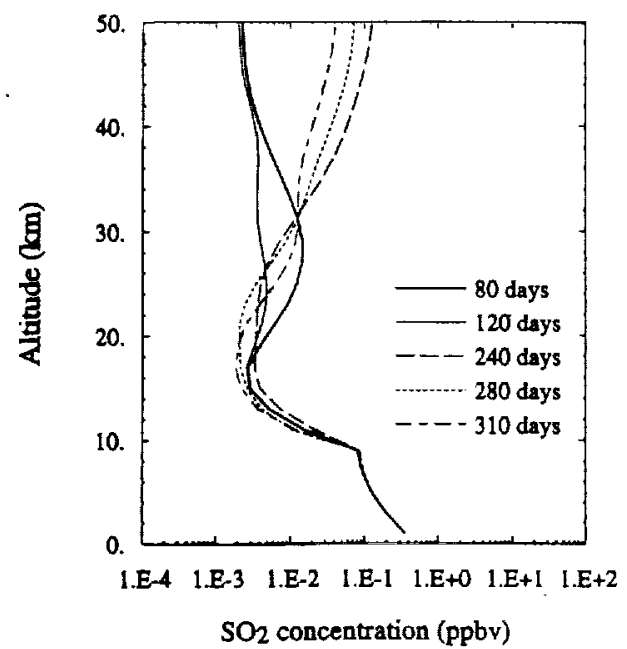

Figure 14. Vertical profiles of $\mathrm{SO}_{2}$ mixing ratio (ppbv) at different times of the year for the case with subsidence.

centration near the evaporation level at $35 \mathrm{~km}$ (Figure 15) is quite sensitive to local temperature variation. A clear correlation between fluctuations in the temperature and simulated $\mathrm{CN}$ is seen during springtime.

The simulated aerosol size distributions for the case with subsidence reveal the detailed evolution of these aerosols (Figure 17). The aerosol size distribution in winter is similar to the ambient size distribution, as might be expected, although the size spectrum shifts to smaller-sized concentrations as some of the bigger particles fall out. Ten days after sunrise, however, a large number of very small $\mathrm{H}_{2} \mathrm{SO}_{4} / \mathrm{H}_{2} \mathrm{O}$ particles are generated by homogeneous nucleation, and the size distribution is bimodal. Unlike the baseline case, the aerosol size spectra for days 50 and 80 (after sunrise) indicate that the small particles have grown to larger sizes, and the typical particle radius on day 80 is at $0.01 \mu \mathrm{m}$.

Figure 18 shows the complete evolution of the $\mathrm{CN}$ distribution from sunset through the end of the year at altitudes from 10 to $60 \mathrm{~km}$. Clearly, a significant enhancement in the $\mathrm{CN}$ concentration occurs during spring (September, October, and November) after sunrise. The peak in the CN layer

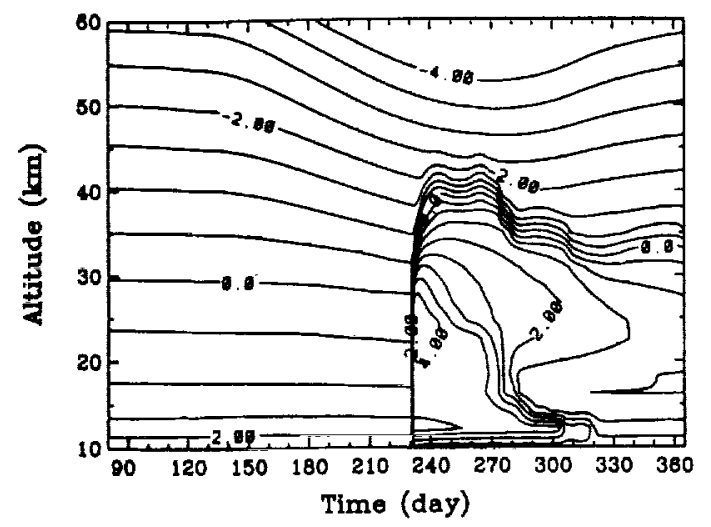

Figure 15. Variation of total aerosol concentration (number per cubic centimeter) with time and altitude in the experiment with a subsiding motion. The concentration contours are shown with the logarithm of the concentrations. The contour interval is $0.5 \mathrm{log}$ units.

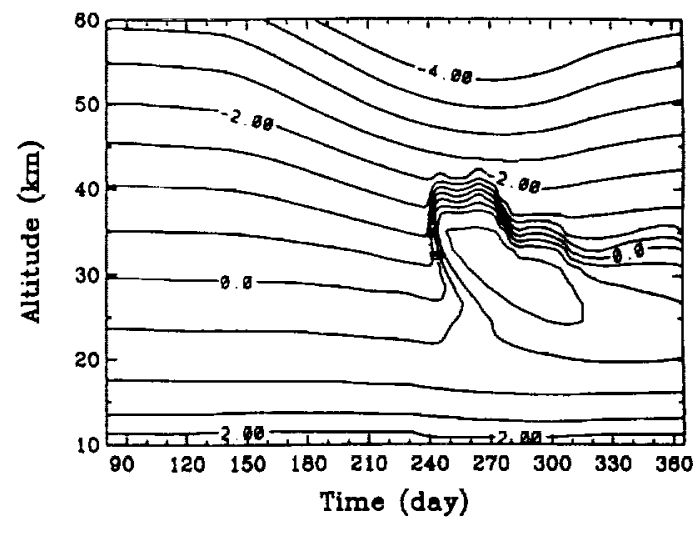

Figure 16. Variation of $\mathrm{CN}$ concentration (number per cubic centimeter) with time and altitude in the experiment with a subsiding motion. The $\mathrm{CN}$ concentrations are shown as isopleths of constant concentration to logarithm. The contour interval is $0.5 \mathrm{log}$ units.

moves from the highest altitude early in spring down to $23 \mathrm{~km}$ in October. The maximum concentration of $\mathrm{CN}$ particles reaches $40 \mathrm{~cm}^{-3}$, with the main layer located between 18 and $37 \mathrm{~km}$. Below $15 \mathrm{~km}, \mathrm{CN}$-sized particles are lost by coagulating with the larger number of ambient aerosols. Above $37 \mathrm{~km}$, higher temperatures cause the volatile $\mathrm{CN}$ droplets to evaporate. The simulated $\mathrm{CN}$ layer is consistent with measurements of the intensity, altitude range, and time of occurrence.

In the simulations, subsiding air carries higher mixing ratios of $\mathrm{SO}_{2}$ from the mesosphere into the stratospheric polar vortex thereby creating a $\mathrm{CN}$ layer, which seems to provide a reasonable explanation for $\mathrm{CN}$ layer formation and the springtime aerosol hole. The case with subsidence also reproduces the observed major features and evolution of the polar $\mathrm{CN}$ layer. We conducted a series of sensitivity experiments with different subsidence velocities and mesospheric $\mathrm{SO}_{2}$ mixing ratios. The intensity of the $\mathrm{CN}$ layer increases with higher $\mathrm{SO}_{2}$ mixing ratios and rates of descending air. It

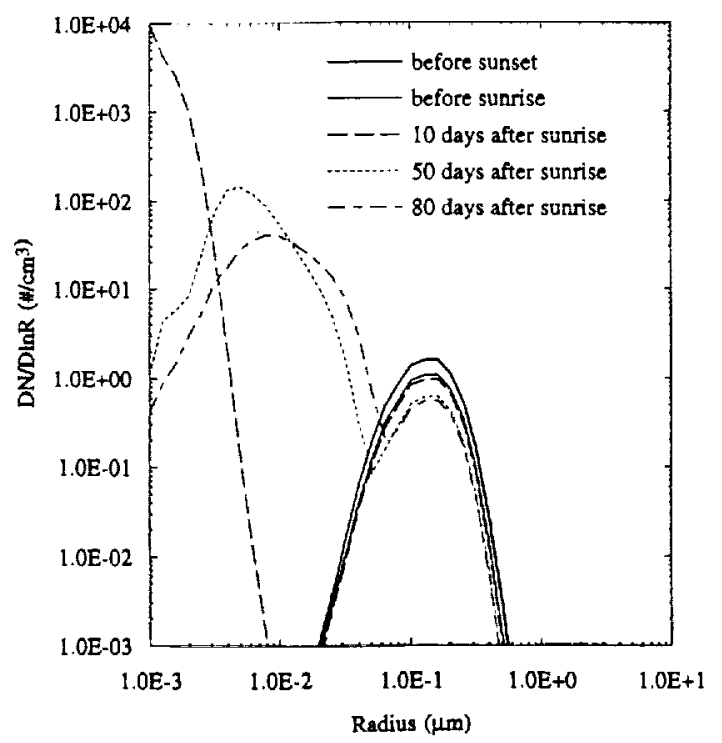

Figure 17. Aerosol size distributions at different times in the experiment with a subsiding motion. 


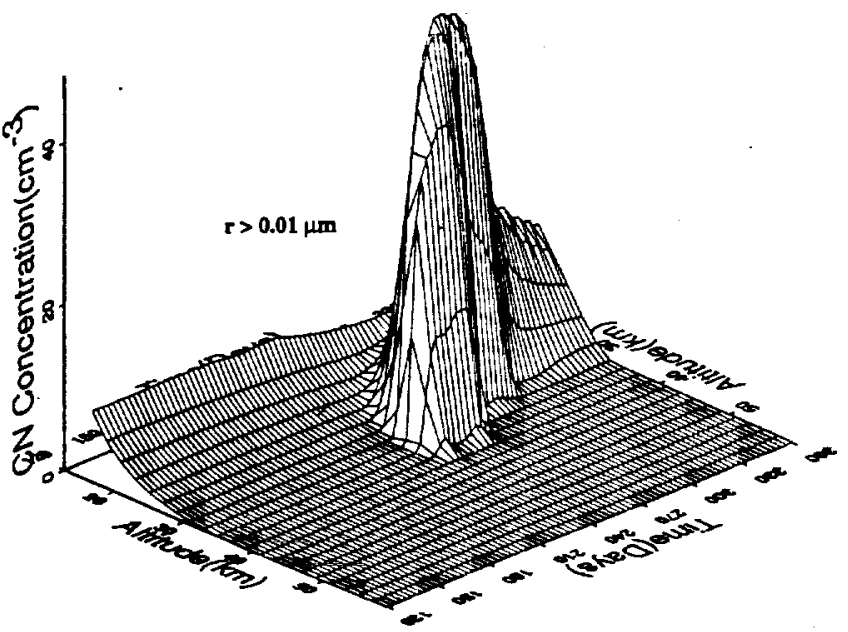

Figure 18. Vertical and temporal distribution of $\mathrm{CN}$ concentration from days 120 to 360 at altitudes between 15 and 59 in the simulation with subsidence.

is found that with an $\mathrm{SO}_{2}$ mixing ratio of 0.1 ppbv or larger, a parameter $\alpha$ in (1) of $0.4 \sim 0.5$ and a velocity $w_{0}$ in the range $0.4 \sim 0.5 \mathrm{~cm} \mathrm{~s}^{-1}$, the generated $\mathrm{CN}$ layers are similar to those observed. According to the observational data from Toon et al. [1989] the vertical velocity near $18 \mathrm{~km}$ is about $-85 \mathrm{~m} \mathrm{~d}^{-1}\left(-0.1 \mathrm{~cm} \mathrm{~s}^{-1}\right)$. The range of maximum subsidence rates near $18 \mathrm{~km}$ used in the model is between 0.02 and $0.1 \mathrm{~cm} \mathrm{~s}^{-1}$ which is close to the observations.

We also run the case with no $\mathrm{SO}_{2}$ but 0.1 ppbv $\mathrm{H}_{2} \mathrm{SO}_{4}$ mixing ratio at the model upper boundary. We found that the simulated CN layer is very weak; the peak concentration is less than $10 \mathrm{~cm}^{-3}$ in this simulation. Moreover, the strength of the layer is not sensitive to increasing the $\mathrm{H}_{2} \mathrm{SO}_{4}$ concentration at the upper boundary. The lack of sensitivity occurs because $\mathrm{H}_{2} \mathrm{SO}_{4}$ vapor can condense on preexisting particles as it is transported from high altitudes to the polar stratosphere. The short lifetime of $\mathrm{H}_{2} \mathrm{SO}_{4}$ vapor prevents its accumulation in the region where $\mathrm{CN}$ events occur. In contrast, the $\mathrm{SO}_{2}$ is not destroyed in the polar night and therefore can serve as a sulfur reservoir for the $\mathrm{CN}$ formation in springtime.

\section{Modified Photochemistry}

To test the importance of oxidation of $\mathrm{OCS}$ by $\mathrm{OH}$ radicals originally proposed by Oppenheimer [1987] and other related chemical processes, the following photochemical reactions involving OCS were considered.

$\left(J_{1}\right) \mathrm{OCS}+h v \rightarrow \mathrm{S}+\mathrm{CO}$

$\left(R_{6}\right) \mathrm{O}+\mathrm{OCS} \rightarrow \mathrm{SO}+\mathrm{CO}, \quad K_{[\mathrm{O}]}-10^{-14}\left(\mathrm{~cm}^{3} \mathrm{~s}^{-1}\right)$

$\left(R_{7}\right) \mathrm{OH}+\mathrm{OCS} \rightarrow \mathrm{SH}+\mathrm{CO}_{2}, K_{[\mathrm{OH}]} \sim 10^{-15}\left(\mathrm{~cm}^{3} \mathrm{~s}^{-1}\right)$

$\left(R_{8}\right) \mathrm{ClO}+\mathrm{OCS} \rightarrow$ products, $K_{[\mathrm{ClO}]}<10^{-16}\left(\mathrm{~cm}^{3} \mathrm{~s}^{-1}\right)$

$\left(R_{9}\right) \quad \mathrm{NO}_{3}+\mathrm{OCS} \rightarrow$ products, $K_{\left.\mathrm{NO}_{3}\right]}<10^{-15}\left(\mathrm{~cm}^{3} \mathrm{~s}^{-1}\right)$

$\left(R_{10}\right) \mathrm{Cl}+\mathrm{OCS} \rightarrow \mathrm{SCl}+\mathrm{CO}, \quad K_{[\mathrm{Cl}]}<10^{-16}\left(\mathrm{~cm}^{3} \mathrm{~s}^{-1}\right)$
The first two reactions are included in the baseline model. As we noted earlier, the photolysis rate of $\operatorname{OCS}\left(J_{1}\right)$ is too small in polar spring to provide an adequate sulfur source for the observed CN layer. An OCS photodecomposition rate, $l_{\text {OCS }}$, of roughly $10^{-7} \mathrm{~s}^{-1}$ is necessary to create a realistic $\mathrm{CN}$ layer. At $25 \mathrm{~km}$, the reaction rate with atom oxygen, $l_{6}=$ $R_{6}[\mathrm{O}]$ is about $10^{-8}$ (if [O] $-10^{6} \mathrm{~cm}^{-3}$ [Brasseur and Solomon, 1986]. This is obviously too slow. The reaction of OH with OCS $\left(R_{7}\right)$ suggested by Oppenheimer [1987], with an upper limit concentration for $\mathrm{OH}$ of $\sim 10^{7} \mathrm{~cm}^{-3}$, is again too small $\left(l_{\mathrm{OH}}-10^{-8} \mathrm{~s}^{-1}\right)$. Measurements show that $\mathrm{ClO}$ mixing ratios in the lower polar stratosphere during spring can exceed 1 ppbv, more than 100 times greater than nomal background stratospheric concentrations [de Zafra et al., 1987; Solomon et al., 1987]. Thus the OCS lifetime against decomposition by reaction $R_{8}$ could become as large as about $10^{-7} \mathrm{~s}^{-1}$, which is an order of magnitude greater than reaction $l_{6}$. This chemical reaction may contribute to the conversion of OCS to $\mathrm{H}_{2} \mathrm{SO}_{4}$ vapor. $R_{9}$ and $R_{10}$ would be more than an order of magnitude less important than $R_{8}$, because the concentrations of $\mathrm{NO}_{3}$ and $\mathrm{Cl}$ are so low.

A ClO concentration field is constructed according to observational data from de Zafra et al. [1987] and Solomon et al. [1987] as

$$
[\mathrm{ClO}]=c(z) \cos \left[\pi\left(\frac{t-240}{60}\right)\right], 230<t<270
$$

where

$$
c(z)= \begin{cases}\exp \left[-\frac{(z-20)^{4}}{2}\right], & z<20 \mathrm{~km} \\ \exp \left[-\left(\frac{z-20}{2}\right)^{2}\right], & z>20 \mathrm{~km}\end{cases}
$$

Here $t$ is time (day) and [ $\mathrm{ClO}]$ is $\mathrm{ClO}$ mixing ratio (ppbv).

When we incorporate reaction $R_{8}$ in the baseline model with the $\mathrm{ClO}$ concentrations shown in Figure 19 and the upper limit (room temperature) rate coefficient of $10^{-16} \mathrm{~cm}^{3}$ $\mathrm{s}^{-1}, \mathrm{CN}$ layers are formed between 15 and $30 \mathrm{~km}$ with peak concentrations of more than $100 \mathrm{~cm}^{-3}$ (Figure 20). This surplus of $\mathrm{CN}$ reflects particle formation, condensation, and coagulation in the presence of abundant $\mathrm{H}_{2} \mathrm{SO}_{4}$ vapor generated by the decomposition of OCS.

Among the photochemical processes listed above, reaction $R_{8}$ is the most likely to provide sufficient $\mathrm{H}_{2} \mathrm{SO}_{4}$ vapor for $\mathrm{CN}$ formation in polar spring. The reaction rate coefficient, however, is probably much smaller than the room temperature upper limit [DeMore et al., 1990] used in the simulation. At much lower stratospheric temperatures than the room temperature the rate coefficient may be 1 to 2 orders of magnitude smaller. Further experimental studies of this mechanism are needed to eliminate this possibility.

\section{Effect of Micrometeorites}

To study the impact on $\mathrm{CN}$ layer formation of an additional particle source associated with micrometeorites, we also did some experiments which include a constant flux of small particles at the upper boundary according to the results from Hunten et al. [1980] and Turco et al. [1980]. These fine meteoritic aerosols slowly migrate downward by diffusion in 

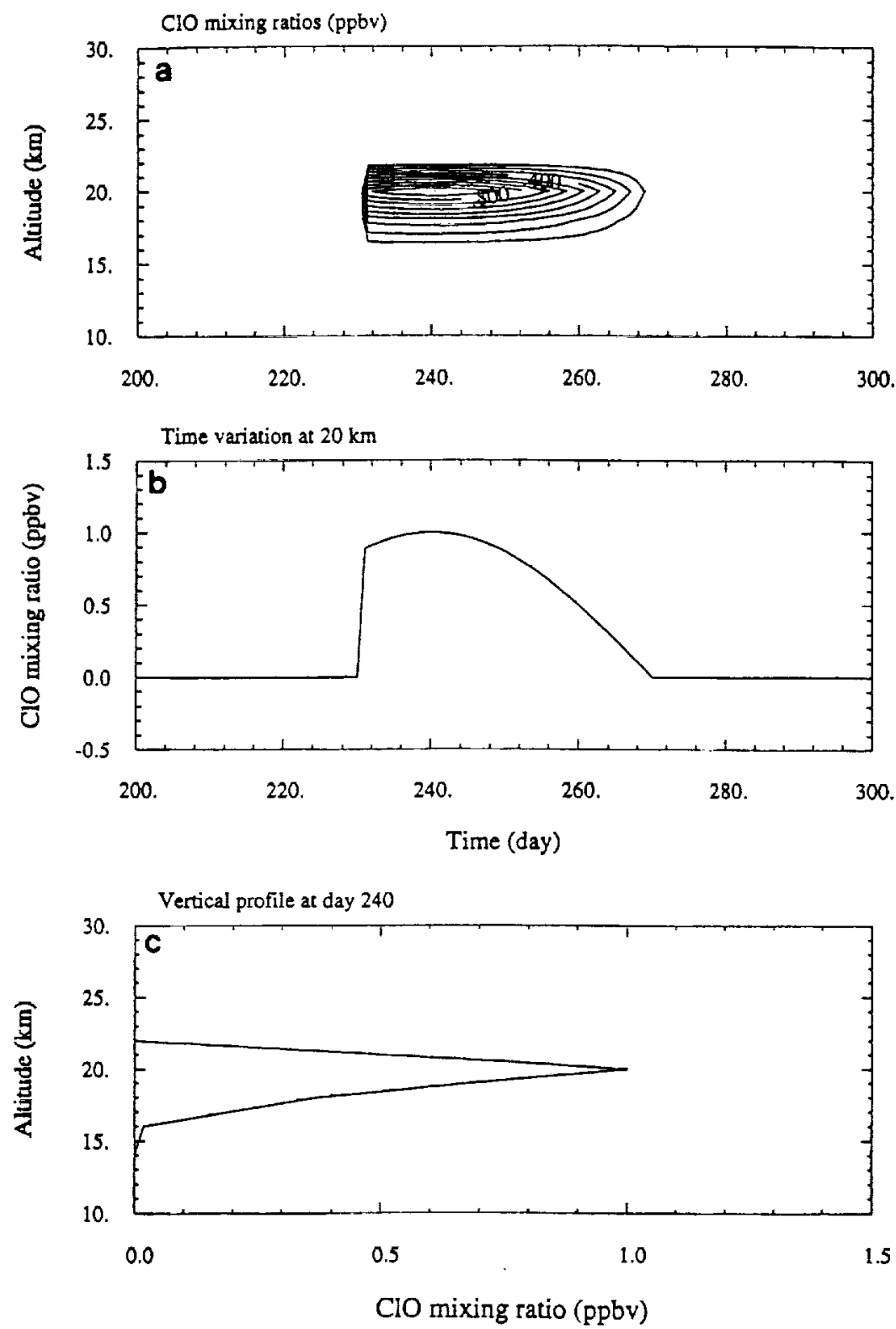

Figure 19. (a)-(c) ClO mixing ratios used in the numerical experiment with reaction $R_{8}$ set to its upper limit value. The enhanced $\mathrm{ClO}$ concentrations were constructed using equation (7) referring to the measurements from de Zafra et al. [1992].

the one-dimensional simulation. Figure 21 shows the steady state aerosol number size distributions obtained by integrating the model 300 days under conditions corresponding to the background state with a meteoritic aerosol source at the upper boundary. Even though there are a larger number of meteoritic dust particles throughout the upper stratosphere, these do not influence $\mathrm{CN}$ concentrations. The aerosol size spectra (Figure 21) show a micrometeorite mode below a $0.01-\mu \mathrm{m}$ radius, with a typical radius of $0.003 \mu \mathrm{m}$. The initial size of the meteoric dust particles, although quite uncertain, are very unlikely to be larger than $0.01 \mu \mathrm{m}$ [Hunten et al., 1980]. Accordingly, micrometeoritic particles themselves are unlikely to change the $\mathrm{CN}(r>0.01 \mu \mathrm{m})$ concentration, or to form a distinct $\mathrm{CN}$ layer.

The micrometeoritic particles were incorporated in the $\mathrm{CN}$ simulation as an initial distribution (Figure 21) in equi- librium with a fixed flux at the upper boundary. Other conditions were similar to those in the baseline experiment. The resultant $\mathrm{CN}$ concentrations, including the vertical distributions and temporal evolution, are almost the same as in the baseline model. When micrometeorites are coupled with either subsiding motion or reaction $R_{B}$ in the model, minor variations in $C N$ concentrations occurred when heterogeneous nucleation was assumed to have a contact angle of $50^{\circ}$ (moderate nuclei). The overall features of the $\mathrm{CN}$ layers remained unchanged. Clearly, the influence of micrometeorites is a secondary factor in $\mathrm{CN}$ layer formation.

We also considered an extreme case by assuming (1) all the micrometeorites serve as very efficient condensation nuclei for sulfate aerosols and are nucleated in a very short time once $\mathrm{H}_{2} \mathrm{SO}_{4}$ vapor is supersaturated and (2) homogeneous nucleation is switched off so that no new particles are 
generated. When we incorporated conditions $I$ and 2 into the model with otherwise the same conditions as in baseline experiment, the model results are almost the same as those in baseline experiment. A CN layer still does not appear. Even if the micrometeorites are assumed to be excellent nuclei for sulfuric acid and water solutions, they did not enhance $\mathrm{CN}$ formation in this experiment.

When conditions 1 and 2 are combined with vortex subsidence and sulfur flux at the upper boundary of the model, a $\mathrm{CN}$ layer is produced similar to that shown in Figure 12. However, the scenario of $\mathrm{CN}$ formation is distinctly different from that in the similar case without meteorites. In the case illustrated in Figure 12 the new sulfuric acid/water droplets are produced by homogeneous nucleation. In contrast, in this case, it is the heterogeneous nucleation of sulfuric acid/water solutions on these preexisting micrometeorites that is the key mechanism to initialize the $\mathrm{CN}$ formation. As we can see in Figure 21, the concentrations of micrometeorites can be more than $100 \mathrm{~cm}^{-3}$ above $30 \mathrm{~km}$. These micrometeorites are heterogeneously nucleated and grow to $\mathrm{CN}$ sizes $(>0.01 \mu \mathrm{m})$ by condensation as they are transported to the lower stratosphere by subsidence.

According to the results from numerous numerical experiments we found that if sufficient concentrations of wettable micrometeorites exist, we can not rule out the possibility of $\mathrm{CN}$ layer formation through heterogeneous nucleation of sulfuric acid and water solutions on these meteorites. However, the presence of sufficient $\mathrm{H}_{2} \mathrm{SO}_{4}$ vapor concentrations is the most crucial factor controlling the occurrence of $\mathrm{CN}$ events. With the subsidence supplying the extra sulfur from the mesosphere, a CN layer can be generated either through homogenous or heterogeneous nucleation, or by both.

\section{Influence of Polar Stratospheric Clouds (PSCs)}

It has been proposed that the largest sulfate particles in the stratosphere may freeze and serve as condensation nuclei for PSCs. The removal of aerosols from the polar vortex may be related to PSC formation in winter and early spring when temperatures drop below about $193^{\circ}-195^{\circ} \mathrm{K}$ (refer to the

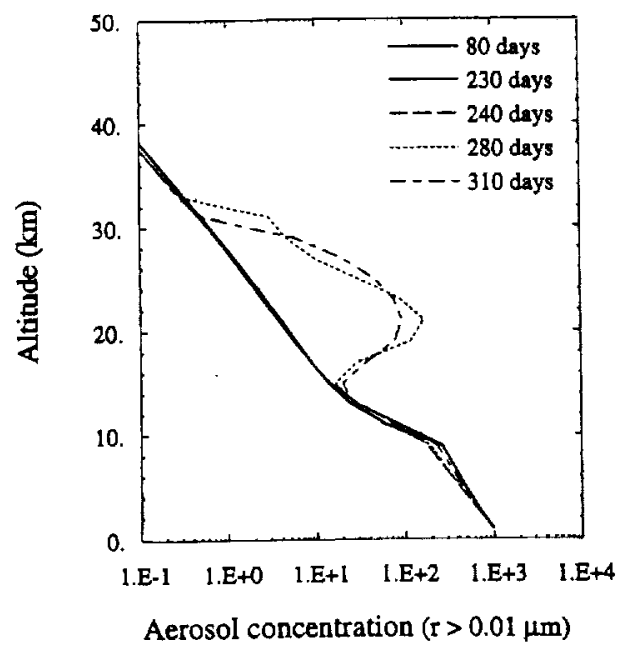

Figure 20. Vertical distributions of $\mathrm{CN}$ concentrations at different times in the numerical experiment with reaction $\boldsymbol{R}_{8}$ set to its upper limit value and elevated $\mathrm{ClO}$ concentrations (see Figure 19).

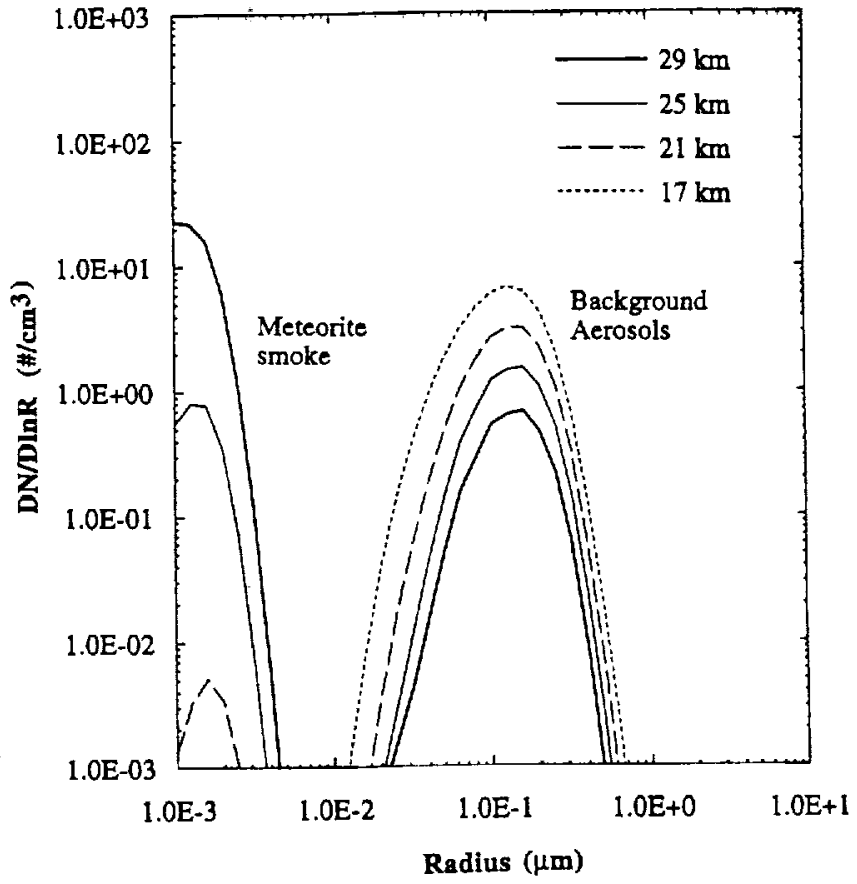

Figure 21. Simulated aerosol size distributions for background conditions including meteoric smoke particles [after Hunten et al., 1980].

temperature variation in Figure 4). The larger PSC particles fall to lower altitudes more quickly than sulfate aerosols and thereby serve as a sink. With the largest sulfate aerosols removed, $\mathrm{H}_{2} \mathrm{SO}_{4}$ vapor loss by condensation on particle surfaces is reduced, which may favor new particle formation by nucleation.

To test the influence of PSCs on $\mathrm{CN}$ formation, it was assumed that whenever the temperature falls below $195^{\circ} \mathrm{K}$, large sulfate particles $(r>2.5 \mu \mathrm{m})$ are scavenged by PSCs. The results suggest that the loss of aerosol surface area due to PSC sedimentation during winter is not a critical mechanism for $C N$ formation. That is, the baseline experiment does not generate a $\mathrm{CN}$ layer even if this process is included; however, when it is combined with vortex subsidence or the $\mathrm{ClO}$ reaction, the predicted $\mathrm{CN}$ layers are slightly intensified.

\section{Summary and Discussion}

The major conclusions derived from numerous model experiments regarding polar $\mathrm{CN}$ layer formation are summarized in Table 2. We found that the variations in photochemical reactions corresponding to the conditions of polar twilight and low temperature control the first appearance of new particle formation. Specifically, $\mathrm{OH}$ radicals play an important role in producing sulfuric acid vapor from $\mathrm{SO}_{2}$, and low temperatures are crucial for homogeneous nucleation, which generates large numbers of small $\mathrm{H}_{2} \mathrm{SO}_{4} / \mathrm{H}_{2} \mathrm{O}$ particles. However, If OCS photolysis is the only mechanism considered, the newly formed particles cannot grow to observable $\mathrm{CN}$ sizes because of the shortage of $\mathrm{H}_{2} \mathrm{SO}_{4}$ vapor. Not only is the photodissociation rate of OCS too small under the weak solar illumination at large zenith angles in early spring 
Table 2. Summary of the Importance of Various Mechanisms for CN Production

\begin{tabular}{|c|c|}
\hline Mechanism & Contribution to CN Layer Formation \\
\hline Low temperatures & essential (initiates homogeneous nucleation) \\
\hline OCS photolysis & secondary (supplies some $\mathrm{H}_{2} \mathrm{SO}_{4}$ vapor) \\
\hline Vortex dynamics & essential (provides high $\mathrm{SO}_{2}$ mixing ratios through subsidence) \\
\hline Oxidation of OCS by $\mathrm{OH}$ & unimportant (unless $\mathrm{OH}$ concentrations exceed $10^{7} \mathrm{~cm}^{-3}$ ) \\
\hline Reaction of OCS with $\mathrm{ClO}$ & possibly unimportant (unless the reaction has upper limit value and a small action energy) \\
\hline Micrometeorite influx & possibly important (if sufficient concentrations exist and are active as condensation nuclei) \\
\hline PSC scavenging & minor (except perhaps in the Antarctic vortex where substantial PSC-induced sulfate sedimentation may occur) \\
\hline
\end{tabular}

$\mathrm{CN}$, condensation nuclei; OCS, carbonyl sulfide; PSC, polar stratospheric cloud.

but also the OCS concentrations would be suppressed by subsidence in the polar vortex over the winter season.

Air descending from the mesosphere in the polar vortex carries higher $\mathrm{SO}_{2}$ mixing ratios into the stratosphere and provides an extra sulfur source for the formation of a $\mathrm{CN}$ layer. In a one-dimensional model, realistic $\mathrm{CN}$ layers are produced when the vortex dynamical process is included. The major features of the $\mathrm{CN}$ layer observed by balloonborne instruments are accurately depicted in the "subsidence" experiment. For instance, the layer appears at higher altitudes in early austral spring and shifts downward to near $25 \mathrm{~km}$ by October. The layers are confined to altitudes between 18 and $37 \mathrm{~km}$. Below $18 \mathrm{~km}$, the ambient particles absorb sulfuric acid vapor fast enough to prevent new $\mathrm{CN}$ production. Above $37 \mathrm{~km}$ the local temperatures are high enough to prevent $\mathrm{H}_{2} \mathrm{SO}_{4}$ vapor from nucleating. The subsidence experiment also explains the small concentrations of optically active aerosols during winter and spring resulting from the intrusion of aerosol-free air from high altitudes. Polar subsidence and photochemistry of $\mathrm{SO}_{2}$ seems to be the most plausible explanation for $\mathrm{CN}$ layer formation in polar spring. Our calculations are based on the parameters such as temperature, subsidence, and solar illumination for the Antarctic stratosphere, but these conditions are similar over Arctic. We believe $\mathrm{CN}$ events in both hemispheres have similar formation mechanisms.

By analyzing all of the reactions involving stratospheric OCS that might initiate sulfate aerosol production in spring, we have found that none of these reactions are likely to provide sufficient $\mathrm{H}_{2} \mathrm{SO}_{4}$ vapor for $\mathrm{CN}$ layer formation and persistence. The photochemical production of $\mathrm{SO}_{2}$ by the reaction between OCS and ClO seems to be a secondary process at best. The OCS $+\mathrm{ClO}$ reaction, although quite uncertain in the absence of sufficient laboratory data, would, at its upper limit room temperature value, be barely adequate to generate a weak $\mathrm{CN}$ layer.

Other sensitivity tests were also conducted. We conclude, for example, that micrometeorites may cause minor variations in $\mathrm{CN}$ concentrations. Heterogeneous nucleation on micrometeorites could be important for $\mathrm{CN}$ formation if the micrometeorites are good condensation nuclei for sulfuric acid and water solutions and have sufficient concentrations in the stratosphere. Homogeneous nucleation in most cases appears to be necessary to explain the increase in $\mathrm{CN}$ concentrations. Indeed, the total sulfate mass in the $\mathrm{CN}$ layer implies such a large $\mathrm{H}_{2} \mathrm{SO}_{4}$ vapor production rate that homogeneous nucleation at polar temperature seems unavoidable. Reductions in the background sulfate aerosol surface area caused by PSC condensation and sedimentation can help $\mathrm{CN}$ layer formation according to our model simu- lations. However, PCS effects cause minor variations in $\mathrm{CN}$ layer properties and cannot explain the appearance of the layer or its overall characteristics.

Acknowledgments. This work was partially supported by NSF grant ATM-89-11836 and NASA grant NAG-1-1126. We used the facility at NASA Ames and San Diego Supercomputer Center for computations.

\section{References}

Brasseur, G., and S. Solomon, Aeronomy of the Middle Atmosphere. D. Reidel, Norwell, Mass., 1986.

Crutzen, P. J., The possible importance of OCS for the sulfate layer of the stratosphere, Geophys. Res. Lett., 3, 73-76, 1976.

DeMore, W. B., S. P. Sander, D. M. Golden, M. J. Molina, R. F. Hampson, M. J. Kurylo, C. J. Howard, and A. R. Ravishankara, Chemical kinetics and photochemical data for use in stratospheric modeling, JPL Publ. 90-1, 217 pp., Jet Propul. Lab., Pasadena, Calif., 1990.

de Zafra, R. L., M. Jaramillo, A. Parrish, P. M. Solomon, B. Connor, and J. Barret, High concentrations of chlorine monoxide at low altitudes in the Antarctic spring stratosphere, I, Secular variation, Nature, 328, 408-41I, 1987.

Fisher, M., A. O'Neill, and R. Sutton, Rapid descent of mesospheric air into the stratospheric polar vortex, Geophys. Res. Letl., 20, 1267-1270, 1993.

Hamill, P., O. B. Toon, and R. P. Turco, Aerosol nucleation in the winter Arctic and Antarctic stratospheres, Geophys. Res. Lett., $17,417-420,1990$.

Hofmann, D. J., Balloon-borne measurements of middle atmosphere aerosol and trace gases in Antarctica, Rev. Geophys., 26, 113-130, 1988.

Hofmann, D. J., Measurement of the condensation nuclei profile to $31 \mathrm{~km}$ in the Arctic in January 1989 and comparisons with Antarctica measurements, Geophys. Res. Lett., 17, 357-360, 1990.

Hofmann, D. J., and J. M. Rosen, Balloon observations of the time development of the stratospheric aerosol event of 1974-1975, $J$. Appl. Meteorol., 17, 56-62, 1977.

Hofmann, D. J., and J. M. Rosen, Stratospheric sulfuric acid fraction and mass estimate for the 1982 volcanic eruption of $\mathrm{El}$ Chichón, Geophys. Res. Letr., 10, 313-316, 1983.

Hofmann, D. J., and J. M. Rosen, Antarctic observations of stratospheric aerosol and high altitude condensation nuclei following the El Chichon eruption, Geophys. Res. Lett., 12, 13-16, 1985.

Hofmann, D. J., J. M. Rosen, J. W. Harder, Aerosol measurements in the winter/spring Antarctic stratosphere, 1, Correlate measurements with ozone, J. Geophys. Res., 93, 665-676, 1988.

Hofmann, D. J., J. M. Rosen, J. W. Harder, and J. V. Hereford, Balloonborne measurements of aerosol condensation nuclei, and cloud particles in the stratosphere at McMurdo Station, Antarctica during the spring of 1987, J. Geophys. Res., 94, 11,253$11,269,1989$.

Hunten, D. M., R. P. Turco, and O. B. Toon, Smoke and dust particles of meteoric origin in the mesosphere and stratosphere, $J$. Almos. Sci., 37, 1342-1357, 1980. 
McCormick, M. P, C. R. Trepte, and G. S. Kent, Spatial changes in the stratospheric aerosol associated with the north polar vortex, Geophys. Res. Lett., 10, 941-944, 1983.

Möhler, $O$., and F. Arnold, Gaseous sulfuric acid and sulfur dioxide measurements in the Arctic troposphere and lower stratosphere: Implications for hydroxyl radical abundance, $J$. Geophys. Res., 19, 1763-1766, 1992.

Nankyoku, N., and C. Kansokutai, Antarct. Meteorol. Data, 31, 269-287, 1990.

Oppenheimer, M., A photochemical source for condensation nuclei in the Antarctic circumpolar vortex, Nature, 328, 702-704, 1987.

Rosen, J. M., and D. J. Hofmann, Balloon measurements of condensation nuclei, J. Appl. Meteorol., 16, 52-56, 1977.

Rosen, J. M., and D. J. Hofmann, Unusual behavior in the condensation nuclei concentration at $30 \mathrm{~km}, J$. Geophys. Res., 88 , 3725-3731, 1983.

Russell, J. M., III, A. F. Tuck, L. L. Gordley, J. H. Park, S. R. Drayson, J. E. Harries, R. J. Cicerone, and P. J. Crutzen, HALOE antarctic observations in the spring of 1991, Geophys. Res. Lett., 20, 719, 1993.

Solomon, P. M., B Connor, R. deZafra, A. Parrish, J. Barret, and M. Jaramillo, Observations of high concentrations of chlorine monoxide in the lower stratosphere during Antarctic spring, II, Secular variation, Nature, 328, 411-413, 1987.

Toon, G. C., C. B. Farmer, L. L. Lowes, P. W. Schaper, J.-F., Blavier, and R. H. Horton, Infrared aircraft measurements of stratospheric composition over Antarctica during September 1987, J. Geophys. Res., 94, 16,571-16,596, 1989.

Toon, O. B., R. P. Turco, D. Westphal, R. Malone, and M. S. Lu, A multidimensional model for aerosols: Description of computational analogs, J. Atmos. Sci., 45, 2123-2143, 1988.
Turco, R. P., P. Hamill, O. B. Toon, R. C. Whitten, and C. S. Kiang, A one-dimensional model describing aerosol formation and evolution in the stratosphere, 1, Physical processes and mathematical analogs, J. Atmos. Sci., 36, 699-717, 1979.

Turco, R. P. O B. Toon, P. Hamill, and R. C. Whitten, Effects of meteoric debris on stratospheric aerosols and gases, J. Geophys. Res., 86, 1113-1128, 1981.

Turco, R. P., O. B. Toon, and R. C. Whitten, Stratospheric aerosols: Observation and theory, Rev. Geophys., 20, 233-279, 1982.

Wilson, J. G., M. Loewenstein, D. W. Fahey, B. Gary, S. D. Smith, K. K. Kelly, G. V. Ferry, and K. R. Chen, Observations of condensation nuclei in the airborne Antarctic ozone experiment: Implications for new particle formation and polar stratospheric cloud formation, J. Geophys. Res., 94, 16,437-16,448, 1989.

Zhao, J.-X. Numerical studies of the stratospheric aerosol layer under background and perturbation conditions, Ph.D. dissertation, 218 pp., Univ. of Calif., Los Angeles, 1993.

O. B. Toon, NASA Ames Research Center, Moffett Field, CA 94035 .

R. P. Turco, Department of Atmospheric Sciences, University of California, Los Angeles, CA 90024.

J. Zhao (corresponding author), Department of Meteorology, School of Ocean and Earth Science and Technology, University of Hawaii, HI 96822 .

(Received May 11, 1994; revised November 17, 1994; accepted November 17, 1994.) 
